\title{
Trichosporon asahii Causing Skin Lesions upon Ileostomy and Ureterostomies in an Immunodeficient Patient: A Case Report and a Mini Review of the Literature
}

\section{Stergios Karapsias*}

Clinical Microbiology Laboratory, 251 General Air Force Hospital, Athens, Greece

*Corresponding author: Stergios Karapsias, Clinical Microbiology Laboratory, 251 General Air Force Hospital, Athens, Greece, Tel: 0306983521853; E-mail: kast594594@yahoo.gr

Received date: February 28, 2017; Accepted date: April 12, 2017; Published date: April 19, 2017

Copyright: @ 2017 Karapsias S. This is an open-access article distributed under the terms of the Creative Commons Attribution License, which permits unrestricted use, distribution, and reproduction in any medium, provided the original author and source are credited.

\begin{abstract}
A case of superficial skin lesions due to $T$. asahii is reported, concerning an immune-deficient patient while receiving micafungin. Yet, $T$. asahii is assumed as intrinsically resistant to echinocandines. Thus, compared to echinocandines, azoles should be preferred in antifungal prophylaxis of immunosuppressed patients because they treat a wider variety of opportunistic fungi, including $T$. asahii. Additionally, $T$. asahii should be regarded as a potential pathogen causing skin lesions in immune-depressed or surgical patients.
\end{abstract}

Keywords: Trichosporon asahii; Skin lesions; Immunodeficiency

\section{Introduction}

Trichosporon asahii is basidiomycetous yeast causing various opportunistic infections in immunodeficient patients. The present short review is based upon a case of fungal skin infections due to $T$. asahii in an immunosuppressed patient.

\section{Case Study}

A 70 year old male patient had been admitted in surgical wards for treatment of urine bladder cancer. After surgical removal of urine bladder and part of small intestine, an ileostomy and two ureterostomies were created, which macroscopically appeared inflammatory and consequently were microbiologically examined via cultures. Skin lesions included erythema, edema, locally increased temperature and mild pain. All cultures (ileostomy, two ureterostomies) showed Trichosporon asahii as the responsible pathogen for all superficial skin inflammatory lesions. Susceptibility testing against $T$. asahii was performed by calculating minimum inhibitory concentrations (MIC) to azoles (sensitivity), amphotericin B (sensitivity) and echinocandines (resistance).

Before T. asahii diagnosis was established, the patient had been receiving micafungin $100 \mathrm{mg} \mathrm{X} 1$ iv (an echinocandine) in a preventive antifungal basis due to his immunodeficiency (cancer, chemotherapy, several surgeries). Yet, T. asahii bibliographically presents endogenous resistance to echinocandines and such resistance has been proved by our laboratory, too. So, despite antifungal treatment, superficial surgical wounds (ileostomy, ureterostomies) were opportunistically colonised and finally infected by $T$. asahii. As soon as fungal susceptibility testing was completed, micafungin treatment was replaced by a miconazole nitrate $2 \%$ cream, locally applied upon infected wounds and skin lesions. After five days of topical treatment, T. asahii was eradicated from infected skin wounds.

\section{Discussion}

T. asahii has been been described as a pathogen causing fatal septic shock in a diabetic patient without cancer or neutropenia in the USA [1], nosocomial urinary tract infections in intensive care unit patients in China [2], community urinary tract infections in diabetic patients in India [3] and bacteraemia in a premature neonate in Greece [4]. Yet, $T$. asahii has been reported to cause urinary tract infections in immunocompetent patients in India [5].

Trichosporon species seem to colonize normal perigenital region as well as urine and catheters of hospitalised patients at the Intensive Care Unit in Brazilian hospitals [6]. Though, T. asahii rather prefers to contaminate urine and catheters than perigenital skin [6]. As a consequence of such a contamination following a potential colonization, our rare report -if not the first one- has presented $T$. asahii skin lesions in an immunodeficient operated patient.

T. asahii bibliographically is intrinsically resistant to echinocandines. For that reason, outbreaks of T. asahii infections, including our case, have been reported in immunosuppressed patients while receiving echinocandines $[7,8]$.

In conclusion, $T$. asahii appears mostly as a pathogen in immunodeficient patients. Since echinocandines are ineffective against such opportunistic fungal infections, they shouldn't be used in a preventive antifungal basis. Compared to echinocandines, azoles should be preferred in antifungal prophylaxis because they treat a wider variety of opportunistic fungi, including $T$. asahii and remain more cost-effective, too. In addition, T. asahii should be regarded as a potential pathogen causing skin lesions in wounds of immunodepressed or surgical patients.

\section{References}

1. Ebright JR, Fairfax MR, Vasquez JA (2001) Trichosporon asahii, a nonCandida yeast that caused fatal septic shock in a patient without cancer or neutropenia. Clin Infect Dis 33:e28-e30.

2. Sun W, Su J, Xu S, Yan D (2012) Trichosporon asahii causing nosocomial urinary tract infections in intensive care unit patients: genotypes, 
Citation: Karapsias S (2017) Trichosporon asahii Causing Skin Lesions upon lleostomy and Ureterostomies in an Immunodeficient Patient: A Case Report and a Mini Review of the Literature. J Pigment Disord 4: 263. doi:10.4172/2376-0427.1000263

Page 2 of 2

virulence factors and antifungal susceptibility testing. J Med Microbio 61:1750-1757.

3. Sabharwal ER (2010) Successful management of Trichosporon asahii urinary tract infection with fluconazole in a diabetic patients. Indian J Pathol Microbiol 53:387-388.

4. Panagopoulou P, Evdoridou J, Bibashi E, Filioti J, Sofianou D, et al. (2002) Trichosporon asahii: An unusual cause of invansive infection in neonates. Pediatr Infect Dis J 21: 169-170.

5. Khan MID (2015) Trichosporon asahii urinary tract infection in immunocompetent patients. Med J Armed Forces India 71: 373-376.
6. Messias (2010) Trichosporon species isolated from the perigenital region, urine and catheters of a Brazilian population. Braz J Microbiol 41:628-634.

7. Bayramoglu G, Sonnez M, Tosun I, Aydin K, Aydin F (2008) Breakthrough Trichosporon asahii fungemia in neutropenic patient with acute leukemia while receiving caspofungin. Infection 36:68-70.

8. Fera MT, La Camera E, De Sarro A (2009) New triazoles and echinocandins: Mode of action, in vitro activity and mechanisms of resistance. Expert Rev Anti Infect Ther 7: 981-98. 